\title{
Geometry vs. Paint Models of Lattice Fringe Visibility for FCC Particles
}

\author{
S. Meyer, P. Fraundorf
}

Department of Physics and Astronomy and Center for Nano Science, University of Missouri - St. Louis, One University Place, St. Louis, MO, 63121 USA

Visibility-maps are tools for analyzing lattice fringes in electron phase-contrast [1] and high-angle annular-darkfield [2] TEM images. The fringe visibility map for FCC crystals is a sphere, with various bands corresponding to orientations that result in reflections of the plane-spacings in the image (Fig. 1). Using a lattice fringe visibility map for FCC crystals, we place bounds on the probabilities of seeing any given type of plane spacing in a TEM microscope that can resolve only the largest two of such spacings. By analyzing the geometry of the regions where bands intersect within the asymmetric unit, and using a flat-space approximation to quantify those regions, we will determine probabilities of seeing orientations that produce $\{200\}$ and/or $\{111\}$ fringes in an image. Applications of these results can e.g. help to detect icosahedral twins, and estimate nano-particle fraction-crystalline as discussed elsewhere here.

It is well known that exact area calculations for shapes on the surface of a sphere can be difficult unless they satisfy specific conditions [3]. However, flat space 2-D approximations for these areas are quite accurate in the case of a square, provided the angular distance between parallel sides is less than 1.2 radians. When the square is the intersection of two bands at $90^{\circ}$ angles to each other, this corresponds to each band having a half-width of $\alpha \leq 0.6$. This kind of intersection is present in the FCC fringe visibility map at the $<100>$ zones, where two $\{200\}$ bands intersect. To further simplify matters, we look at only the asymmetric unit of the crystal. On the visibility map, this unit is a spherical triangle with area $=\pi / 12$ steradians. When considering only the two largest spacings which the most common microscopes can resolve, there is only one other intersection type, at the $<110>$ zone where two $\{111\}$ bands intersect with a $\{200\}$ band (Fig. 2). Within the asymmetric unit, only the areas around the $<110>$ zone need to be approximated, with parallelograms and triangles in a flat 2-D geometry. The accuracy of the square intersection has already been verified (Fig. 3). These regions in the $<110>$ zones correspond to seeing cross fringes of equal width at a $70.6^{\circ}$ in an image. Once these areas are calculated, they can be removed from the exact areas for the band regions. The area of any one band around the entire sphere, with half-width $\alpha$, is $4 \pi \sin \alpha$. Dividing this by 48 gives the exact total area of a band within the asymmetric unit. Removing the approximated intersection areas from the total area of the band, and comparing to the total area $\pi / 12$, we can place an upper bound on the probability of seeing single fringes associated with that band. Doing this for only two types of bands, we can analyze the probability of seeing single fringes, cross fringes, or no fringes in an image of randomly oriented particles.

The results we have described are accurate for images of particles with a $t / \Gamma>3 \mathrm{~nm}$, and they are very specific as they only apply to FCC particles, and only when the largest two spacings are visible in a given microscope. Still, the method does well for verification of more robust computer models, such as a paint model (Fig. 4) which is capable of counting color overlap in each pixel of a computer generated image and can be extended to much more complicated fringe visibility maps. 
References:

[1] P. Fraundorf et al., Journal of Applied Physics 98:114308 (2005)

[2] P. Wang et al., Ultramicroscopy 106:277-283 (2006)

[3] Chamberlain and Duquette, Jet Propulsion Laboratory Publication 07-3 (2007)
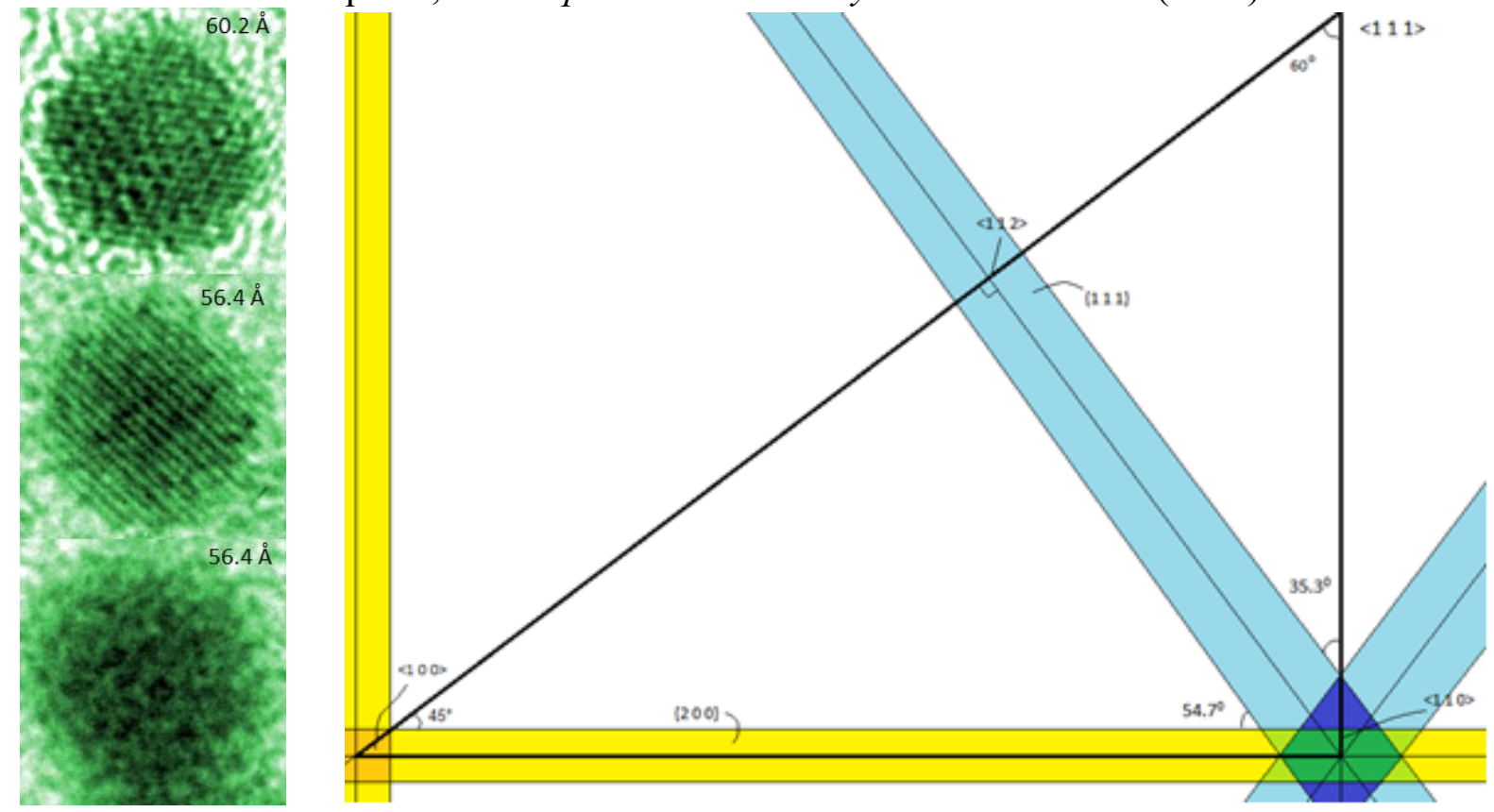

Figure 1. (left) Examples of cross, single, and no fringes shown. Fieldwidths listed on each image.

Figure 2. (right) 2-D schematic of asymmetric unit in FCC map.
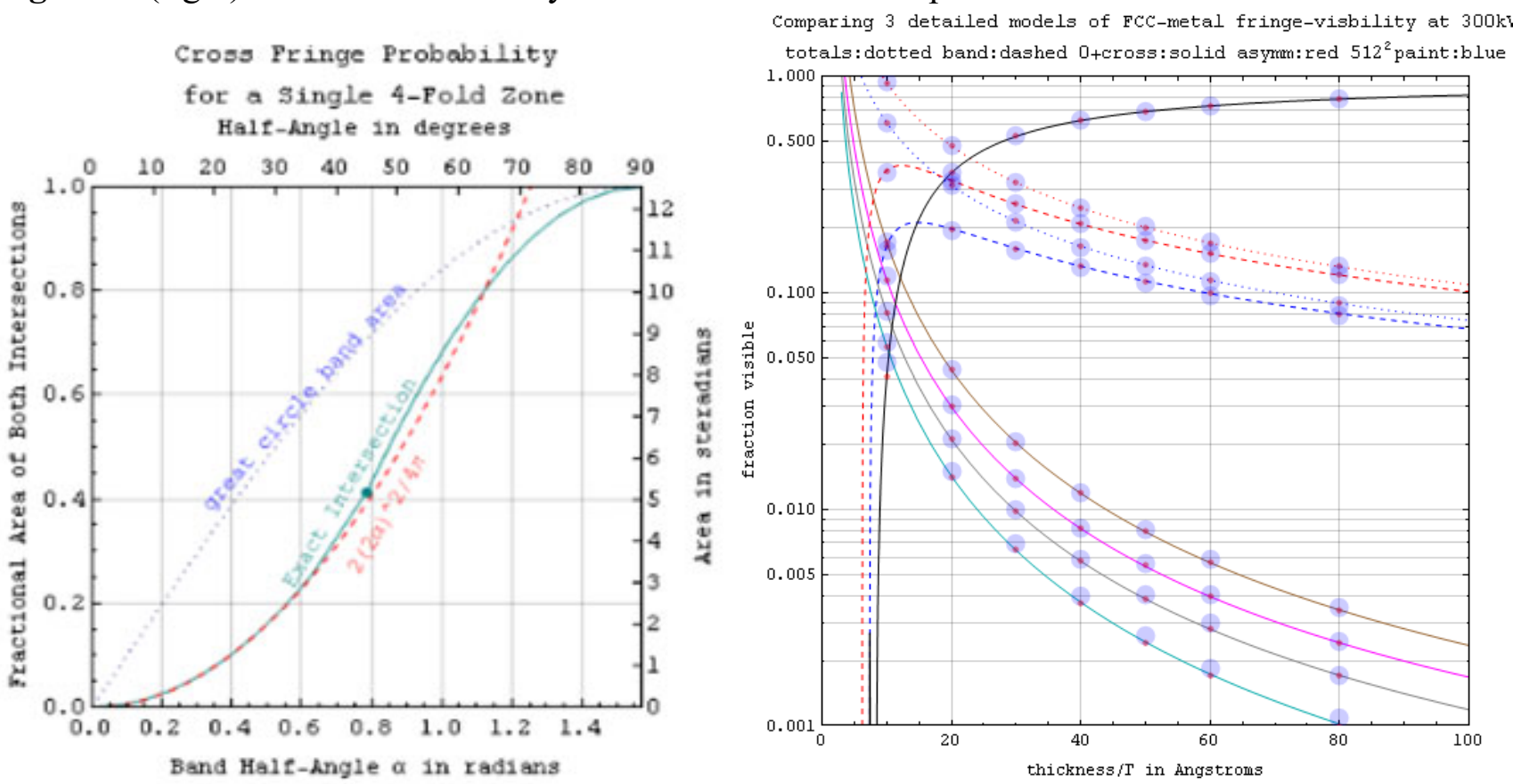

Figure 3. (left) Area of square intersections in cyan, compared with a flat space approximation in red. Figure 4. (right) Comparing 2 geometry and 1 paint visibility model. 